\title{
Photon echo studies of photosynthetic light harvesting
}

\author{
Elizabeth L. Read $\cdot$ Hohjai Lee $\cdot$ Graham R. Fleming
}

Received: 26 November 2008/ Accepted: 21 May 2009/Published online: 10 July 2009

(c) The Author(s) 2009. This article is published with open access at Springerlink.com

\begin{abstract}
The broad linewidths in absorption spectra of photosynthetic complexes obscure information related to their structure and function. Photon echo techniques represent a powerful class of time-resolved electronic spectroscopy that allow researchers to probe the interactions normally hidden under broad linewidths with sufficient time resolution to follow the fastest energy transfer events in light harvesting. Here, we outline the technical approach and applications of two types of photon echo experiments: the photon echo peak shift and two-dimensional (2D) Fourier transform photon echo spectroscopy. We review several extensions of these techniques to photosynthetic complexes. Photon echo peak shift spectroscopy can be used to determine the strength of coupling between a pigment and its surrounding environment including neighboring pigments and to quantify timescales of energy transfer. Two-dimensional spectroscopy yields a frequency-resolved map of absorption and emission processes, allowing coupling interactions and energy transfer pathways to be viewed directly. Furthermore, 2D spectroscopy reveals structural information such as the relative orientations of coupled transitions. Both classes of experiments can be used to probe
\end{abstract}

\footnotetext{
E. L. Read · H. Lee · G. R. Fleming $(\bowtie)$

Department of Chemistry, University of California Berkeley,

221 Hildebrand Hall, Berkeley, CA 94720, USA

e-mail: grfleming@lbl.gov

E. L. Read - H. Lee · G. R. Fleming

Physical Biosciences Division, Lawrence Berkeley National

Laboratory, Berkeley, CA, USA

Present Address:

E. L. Read

Department of Chemical Engineering, Massachusetts Institute

of Technology, 77 Massachusetts Ave, Cambridge, MA 02139, USA
}

the quantum mechanical nature of photosynthetic lightharvesting: peak shift experiments allow quantification of correlated energetic fluctuations between pigments, while 2D techniques measure quantum beating directly, both of which indicate the extent of quantum coherence over multiple pigment sites in the protein complex. The mechanistic and structural information obtained by these techniques reveals valuable insights into the design principles of photosynthetic light-harvesting complexes, and a multitude of variations on the methods outlined here.

Keywords Photon echo - Photon echo peak shift . 2D Fourier transform photon echo spectroscopy . Spectral band broadening - Reaction center - FMO . LH3 - Electronic quantum coherence

$\begin{array}{ll}\text { Abbreviations } & \\ \text { 2D } & \text { Two-dimensional } \\ \text { FMO } & \text { Fenna-Matthews-Olson } \\ \text { PMT } & \text { Photomultiplier tube } \\ \text { 3PEPS } & \text { Three-pulse photon echo peak shift } \\ \text { 1C3PEPS } & \text { One-color three-pulse photon echo peak } \\ & \text { shift } \\ \text { 2C3PEPS } & \text { Two-color three-pulse photon echo } \\ & \text { peak shift } \\ \text { BChl } & \text { Bacteriochlorophyll } \\ \text { Bpheo } & \text { Bacteriopheophytin } \\ \text { Rb. sphaeroides } & \text { Rhodobacter sphaeroides } \\ \text { Rs. rubrum } & \text { Rhodospirillum rubrum } \\ \text { CCD } & \text { Charge-coupled device } \\ \text { LH1 } & \text { Light-harvesting complex 1 } \\ \text { LH2 } & \text { Light-harvesting complex } 2 \\ \text { LH3 } & \text { Light-harvesting complex } 3 \\ \text { LO } & \text { Local oscillator }\end{array}$




\section{Introduction}

The electronic absorption and emission spectra of photosynthetic pigment-protein complexes (Blankenship 2002) are generally broad and lack the kind of details needed to provide insight into their structure, function, and design principles. While the broad absorption bands are advantageous for solar energy absorption, progress in understanding their exquisite effectiveness in light harvesting and trapping, and in charge separation to initiate the chemistry of photosynthesis, requires that we find ways to remove at least some of the broadening that obscures the information content.

What are the origins of the broadening of spectra (Fleming and Cho 1996; Parson 2007) of photosynthetic complexes? In general, there are five factors at work. (1) The bare electronic transitions are broadened by the vibrational transitions (of both chromophore and protein) that accompany them. These transitions arise because the atomic nuclei have different equilibrium positions when the chromophore (e.g., chlorophyll) is in the excited state than in the electronic ground state. This is generally called homogeneous broadening. (2) Individual chromophores in different members of the entire protein ensemble may have slightly different electronic transition energies, even though they occupy structurally identical locations. This can arise because of different ionization states of protein side chains close to their $\mathrm{pK}_{\mathrm{a}}$, different orientations of side chains, slight distortions of the overall protein structure, and a host of similar small influences. The overall effect is to smear out the transition to produce inhomogeneous broadening. (3) Chlorophylls and other pigments are generally bound in a variety of non-equivalent sites in an individual protein complex. For example, the FennaMatthews-Olson (FMO) complex of green sulfur bacteria binds seven bacteriochlorophyll molecules each in a unique site. This type of inhomogeneous broadening may produce a set of more discrete transition energies than the broadening arising by mechanism (2). Both (2) and (3) give transition energies that vary slowly or not at all on the time scale of the optical functions of photosynthetic complexes. (4) In many photosynthetic complexes, the chromophores are held very close to one or more neighbors leading to electronic mixing and associated spectral shifts from the individual molecule's unperturbed transition. This can lead to a set of chemically identical chromophores having a significantly broader spectrum than a similar, but non-interacting, set of molecules. (5) Finally, several processes can, and often do, happen very fast in photosynthetic complexes, leading to lifetime broadening. An excellent summary of the spectroscopy of photosynthetic complexes can be found in Van Amerongen et al. (2000).
Photon echo spectroscopy (Mukamel 1995; Parson 2007) can often remove or greatly diminish the type of broadening described in (2). Indeed, the inhomogeneous broadening can be used to observe the energy flow both within and between photosynthetic complexes. A newly developed form of photon echo spectroscopy, twodimensional Fourier transform photon echo spectroscopy, can be used to unravel the interactions described in (4) as well as remove type (2) broadening, and reveal, on their characteristic timescale, the relaxation pathways within individual complexes and reveal striking details about their design and the origins of their great efficiency. Below, we outline the origins of photon echo (and related) signals and describe a number of photon echo-based experimental techniques applied to problems in photosynthesis.

The basis of photon echo spectroscopy, as with other "ultrafast" techniques, is the interrogation of a system with laser pulses short enough to track dynamical processes of interest. In this work, ultrafast means tens of femtoseconds (where a femtosecond is $10^{-15} \mathrm{~s}$ ), a timescale on which the fastest energy transfer processes occur between neighboring pigments in light-harvesting complexes. The method requires a sequence of laser pulses to interrogate the sample and, as with pump-probe and related experiments, allows observation of excited state dynamics. However, in photon echo experiments, the laser pulse sequence is specifically designed to take advantage of the reversibility of dephasing, illustrated in a general way as follows.

Upon irradiation by a laser pulse, the system begins to oscillate between quantum energy levels. A full quantum mechanical description is beyond the scope of this article, but an analogy can be drawn to a collection of springs, set into motion by the external perturbation (the pulse). Imagine that each of the springs oscillates with a slightly different frequency, analogous to inhomogeneous broadening wherein the electronic transition frequencies of a collection of chromophores vary, described by (2) above for photosynthetic light-harvesting complexes. The result of this distribution of frequencies is that the "springs," oscillating in phase immediately after interaction with the pulse, become gradually less synchronized over time. This is known as dephasing. Imagine then that at some later instant, the motion of the springs is simultaneously reversed by another perturbing pulse. As long as each of the springs maintains its original oscillation frequency and changes only its direction, the overall dephasing is reversed also. When this reverse dephasing or rephasing process occurs not with springs but with a collection of chromophores interacting with laser pulses, the effect is for the sample to emit a light pulse "echoing" the input pulse at the instant when the oscillators are once more in phase. 
The key to the unique information contained in photon echo signals is that the appearance of a photon echo signal depends on each of the springs remembering its initial oscillation frequency and phase. If, on the other hand, the frequencies are individually modified or the phases shifted (as can occur through coupling to vibrational motions of the pigments or proteins), the collective motion of the springs devolves into random noise; the constructive interference-rephasing-is never realized, and a photon echo signal is not emitted. Thus, the signal is uniquely sensitive to the coupling between the electronic transitions on the pigments and the nuclear motions of the "bath" (motions of the pigments themselves and of the surrounding protein). Recent work, including some of the experiments summarized here, has shown that, in fact, the detailed pigment-protein interactions in photosynthesis play an important role in controlling energy flow through the complexes. Furthermore, photon echo signals track energy transfer between the electronic states of neighboring chromophores. Therefore, photon echo experiments are well suited to the study of photosynthetic light harvesting.

The experimental pulse sequence for three-pulse photon echo experiments is shown in Fig. 1. The first input pulse instigates the initial dephasing process described above. The effect of the second pulse is to freeze the "springs," halting dephasing; the third pulse triggers rephasing by setting the motion in reverse. The initial dephasing period, the interval between pulses 1 and 2 , is labeled $\tau$ or the "coherence" time (as the oscillation occurs because the system is in a coherent quantum mechanical superposition state). The second interval is known as $T$ or the "population" time (also called the "waiting" time), and the third, $t$, as the "echo" time. The system's ability to rephase and generate a photon echo diminishes with increasing population time, as each chromophore gradually loses its "memory" of its initial frequency due to interactions with the solvent or energy transfer between pigments. Therefore, experimentally varying $T$ allows experimentalists to observe dynamical processes.

How does the experimentalist enforce the above sequence of events? One way is to experimentally "select" these processes using a noncollinear beam geometry. The geometry varies depending on the experimental scheme, as

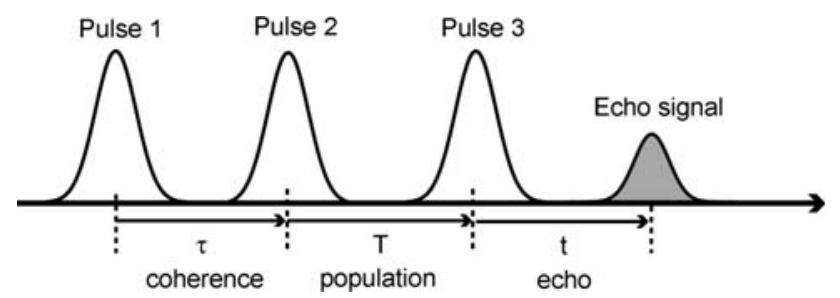

Fig. 1 Pulse sequence for three-pulse photon echo experiment described below. Due to conservation of momentum, the constructive interference of polarization (the photon echo) is scattered in a predictable, so-called phase-matched, direction. Phase matching selects desired signals and allows background-free detection. The use of phase matching is a distinct advantage of optical photon echo techniques over their nuclear magnetic resonance analogs, and is made possible by the large sample size relative to the wavelength of the incident radiation. Detection schemes depend on the particular type of photon echo experiment performed. The experiments illustrated below demonstrate how three-pulse photon echo experiments can be designed to conveniently probe various aspects of the pigmentprotein interactions and energy transfer processes in photosynthesis.

\section{Photon echo peak shift spectroscopy}

\section{Experimental considerations}

Preparing samples of photosynthetic proteins for the study by echo experiments generally requires solubilizing the isolated proteins of interest in a buffer solution with a small amount of detergent. The use of a narrow $(\sim 100-200 \mu \mathrm{m})$ quartz sample cell minimizes effects due to reabsorption of emitted signals. Furthermore, the optical density (OD) of the sample must be chosen to minimize signal distortions due to propagation of signal and pulses through the sample (exacerbated at high ODs) or interference with the solvent response (exacerbated at low ODs) (Christensson et al. 2008). In the experiments presented here, sample ODs were in the range of 0.1-0.3. In high temperature studies, the sample is often flowed through the cell to continuously regenerate fresh sample in the focal spot of the laser beams. However, photon echo experiments are often performed on low temperature glasses to reduce broadening due to the nuclear motions as described above. These samples are prepared by mixing the protein solution with a cryoprotectant such as glycerol (to prevent formation of cracks, which would scatter laser light into the detector) and freezing the sample in a windowed cryostat to the desired temperature. The cryotstat is mounted on a movable stage in the laser beam path, such that the sample may be aligned to the focal point of the laser beams. Localized sample damage is avoided by periodically shifting the cell laterally or vertically to an unused spot and by minimizing the input power of the laser beams as much as possible. Also, at very high excitation energies, it is possible to create multiple excitations (excitons) in the sample and produce spurious signals in the same phase-matched directions as the third order signal. This possibility is discussed by Bruggemann et al. (2007). 
Routine generation of tunable, femtosecond laser pulses using Ti:Sapphire sources has been achieved over the last two decades (Jimenez and Fleming 1996; Demtroder 2003; Rulliere 2003; Parson 2007). In the photon echo experiments described below, three ultrashort pulses are aligned to pass the vertices of an equilateral triangle on a plane perpendicular to pulse propagation and tightly focused on a sample (Fig. 2). Echo signals are generated in phase-matched directions (e.g., $\quad-\mathbf{k}_{1}+\mathbf{k}_{2}+\mathbf{k}_{3}, \quad+\mathbf{k}_{1}-\mathbf{k}_{2}+\mathbf{k}_{3}$, or $+\mathbf{k}_{1}+\mathbf{k}_{2}-\mathbf{k}_{3}$, where the $\mathbf{k s}$ are the momentum vectors of the laser beams). The photon echo signals in selected phase-matched directions are spatially filtered into the detection system by placing a mask after the sample, thereby blocking other signals and scattered light. A photomultiplier tube (PMT) or a photodiode collects the signals. Since the detectors respond more slowly than the experimental time scale, one obtains time $t$-integrated photon echo signals as a function of $\tau$ and $T$.

One-color three-pulse photon echo peak shift (1C3PEPS)

In disordered systems like photosynthetic complexes where electronic dephasing is extremely rapid, it is well established that the photon echo peak shift provides useful information about solvation dynamics, i.e., the rearrangement of the "solvent" (the protein environment) nuclei to accommodate electronic excitations on the chromophores. The peak shift $\left(\tau^{*}\right)$ is defined simply as the coherence time $(\tau)$ at which the photon echo signal reaches maximum intensity for a given $T$. For precise determination of $\tau^{*}$, the average peak shift of echo signals from two different phase matching directions $\left(-\mathbf{k}_{1}+\mathbf{k}_{2}+\mathbf{k}_{3}\right.$ and $\left.+\mathbf{k}_{1}-\mathbf{k}_{2}+\mathbf{k}_{3}\right)$ is often obtained (Fig. 2). The usefulness of 1C3PEPS lies in the fact that it closely follows the time correlation function of a transition frequency of a pigment, which contains solvation dynamics information (Cho et al. 1996). In general, the initial peak shift reflects the system-bath coupling strength because it is proportional to the reciprocal value of

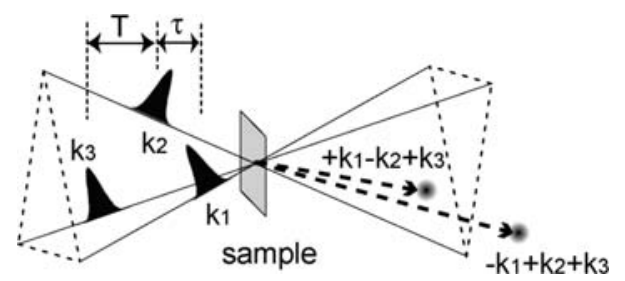

Fig. 2 Three-pulse photon echo peak shift experiment configuration. Three pulses are focused on a sample and the photon echo signals are emitted in the phase-matched direction, $-\mathbf{k}_{1}+\mathbf{k}_{2}+\mathbf{k}_{3}$ and $+\mathbf{k}_{1}-\mathbf{k}_{2}$ $+\mathbf{k}_{3}$. $\lambda_{1}=\lambda_{2}=\lambda_{3}$ for 1C3PEPS, $\lambda_{1}=\lambda_{2}<\lambda_{3}$ for downhill 2C3PEPS, $\lambda_{1}=\lambda_{2}>\lambda_{3}$ for uphill 2C3PEPS, and $\lambda_{1}=\lambda_{3} \neq \lambda_{2}$ for $2 \mathrm{CECPE}$. ks and $\lambda$ s are the momentum vectors and the wavelengths of the pulses, respectively the mean square fluctuation amplitude of the transition frequency. Peak shifts at large $T$ indicate the extent of static disorder, and the decay captures population dynamics. For example, Jimenez et al. (1997) revealed that initial peak shifts for light-harvesting complexes (LH1 and LH2) of purple photosynthetic bacteria, Rhodobacter $(R b$.) sphaeroides are large $(\sim 25 \mathrm{fs})$ compared to the peak shifts of typical dyes in polar solvents (10-15 fs), which indicates weak coupling of the pigments in these complexes to the surrounding protein matrix. This relatively weak coupling may be essential to minimize heat dissipation to the surroundings and, therefore, maximize the energy transfer efficiency from LH2 to LH1 to the reaction center. Another 1C3PEPS experiment on the isolated B820 subunit (a subunit of the LH1 complex, so-called because it absorbs near $820 \mathrm{~nm}$ ) of LH1 in Rhodospirillum rubrum, in comparison with 1C3PEPS on the whole LH1 complex, clearly demonstrated the contribution of energy transfer to the 1C3PEPS signal decay (Fig. 3) (Yu et al. 1997). The signal from the LH1 complex showed a rapid decay component in early $T$ corresponding to energy transfer around the ring and resulting in a small peak shift value at long $T$ (circles). Note that (excitation) energy transfer from one (excited) molecule to another leads to loss of correlation. To the contrary, the energy transfer out of the subunit is blocked in the B820 subunit, which consists only of one $\alpha$ and one $\beta$ transmembrane polypeptide and two BChla molecules. Therefore, the B820 subunit exhibits a generally large peak shift (squares, Fig. 3). The solid line indicates the simulated 1C3PEPS profile with the same parameters for the LH1 complex but without an energy transfer factor. The experiments also demonstrate that the photon echo peak shift is

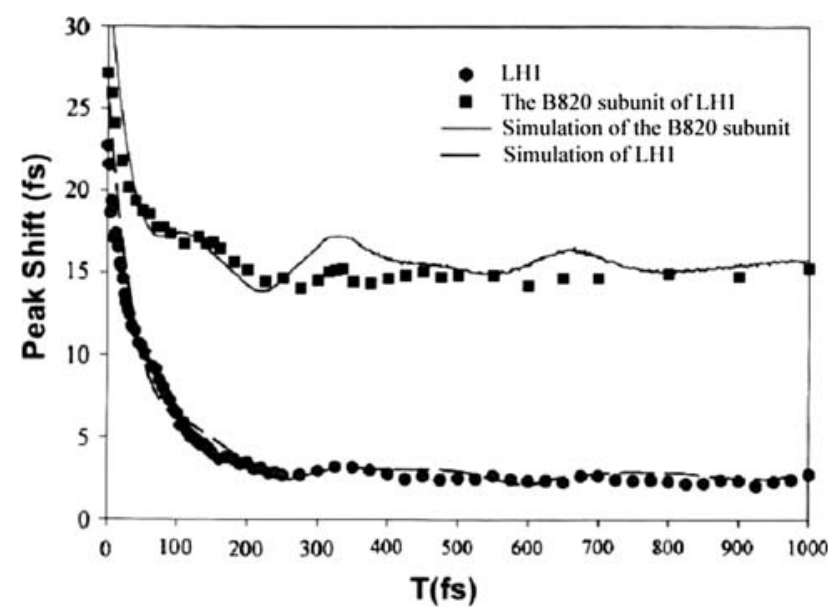

Fig. 3 1C3PEPS measurements of LH1 of Rhodobacter $(R b$.) sphaeroides (circles) and the B820 subunit from LH1 of Rhodospirillum (Rs.) rubrum (squares). The solid lines represent two simulations with identical input parameters except that the energy transfer rate is set to zero for the B820 sample (Yu et al. 1997). Figure reprinted by permission from Elsevier (Yu et al. 1997) 
sensitive to energy transfer within the laser pulse window as well as energy transfer out of the detection window because the peak shift measures the rephasing capability. Moreover, unlike conventional transient absorption or time-resolved fluorescence studies, it is insensitive to reverse energy transfer between transitions of similar energies. These features are useful in studying the diagonal elements of a Hamiltonian of photosynthetic systems in which multiple replicas of pigments are common. In this sense, the evolution of photon echo peak shift reflects excited state dynamics of a photosynthetic system in detail.

Two-color three-pulse photon echo peak shift (2C3PEPS)

Pigments in photosynthetic pigment-protein complexes are often closely packed and form an excitonically coupled system, described as broadening source (4) above and shown schematically in Fig. 4. For instance, the reaction center of the purple bacterium $R b$. sphaeroides has three distinct absorption bands assigned to the $\mathrm{Q}_{\mathrm{y}}$ bands of the primary bacteriochlorophylls (BChls), accessory BChls, and bacteriopheophytins (Bpheos), named the $\mathrm{P}, \mathrm{B}$, and $\mathrm{H}$ bands. However, the excitonically coupled states contain the properties of electronic states from different molecules, and they are correlated to some extent. The notations P, B and $\mathrm{H}$ denote only the major contributing molecules to each state. The recently developed 2C3PEPS method is suitable for investigating such correlated electronic states when the states are of different energies. The more correlation between the states, the larger the peak shift signal

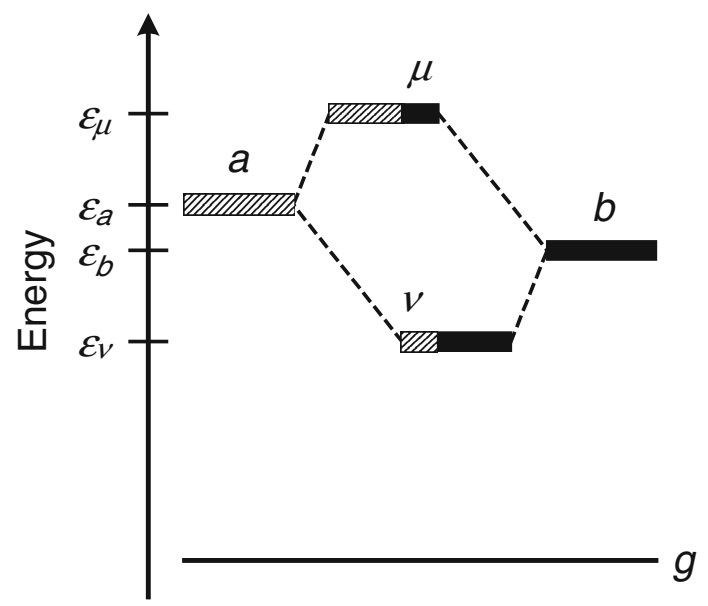

Fig. 4 Energy diagram for an excitonically coupled system. $a$ and $b$ are the localized states of two different chromophores (if the coupling interaction energy were zero), and $\mu$ and $v$ are the upper and lower excitonically coupled states formed from the mixing of $a$ and $b$. Hatched and solid bars depict conceptually the portion of the contribution from $a$ and $b$ individually to the mixed states. Because $\mu$ and $v$ contain character of both $a$ and $b$, they are correlated generated, as an extended concept of 1C3PEPS. In practice, the 2C3PEPS experiment is performed using one wavelength for the first two laser pulses and a different wavelength for the last pulse in a setup similar to that in Fig. 2. If the first two pulses are at a higher energy than the last one, the experiment is called "downhill" and if lower, it is called "uphill." In particular, 2C3PEPS enables us to directly determine the coupling constant, $J$, between the two coupled states, i.e., the off-diagonal elements of the Hamiltonian, without prior knowledge about the site energies of the pigments in the protein matrix. That is, it allows researchers to differentiate between broadening sources (3) and (4) described in the Introduction. $J$ is related to the mixing angle $(\theta)$ by :

$\tan (2 \theta)=\frac{J}{\varepsilon_{a}-\varepsilon_{b}}$,

where $\varepsilon_{a}$ and $\varepsilon_{b}$ are the monomer energies (or site energies in protein matrix) of molecules $a$ and $b$, respectively. The mixing angle can be obtained from the experimental mixing coefficient, $C_{\mu \nu}$ :

$C_{\mu \nu}=2 \sin ^{2} \theta \cos ^{2} \theta \approx \frac{\tau_{\mu \nu}^{*}(T)}{\tau_{\mu \nu}^{*}(T)+\frac{1}{2 \kappa}\left(\tau_{\mu}(T)+\tau_{v}(T) \kappa^{3}\right)}$,

where $\kappa=\tau_{\mu \nu}^{*}(T) / \tau_{v \mu}^{*}(T)$ (Mancal and Fleming 2004). As can be seen in the notation of Fig. $4, \tau_{\mu}^{*}(T)$ and $\tau_{v}^{*}(T)$ represent the 1C3PEPS values for upper and lower excitonic states, respectively, and $\tau_{\mu \nu}^{*}(T)$ and $\tau_{v \mu}^{*}(T)$ represent uphill and downhill 2C3PEPS, respectively. The value of $J$ can be determined using the following equation based on the difference in energy between the two observed exciton states:

$E_{\mu}-E_{v}=2 J \sqrt{1+\left(\frac{1}{\tan (2 \theta)}\right)^{2}}$.

The method was applied to Bpheos ( $\mathrm{H}$ band) and accessory BChls (B band) of the reaction center of $R b$. sphaeroides using pulses centered at 750 and $800 \mathrm{~nm}$, yielding an estimate of the coupling constant between them of $\sim 170 \pm 30 \mathrm{~cm}^{-1}$ (Parkinson et al. 2007).

Another version of the two-color three-pulse photon echo experiment alternates colors in the pulse sequence to generate specific electronic coherences (two-color electronic coherence photon echo, 2CECPE) (Lee et al. 2007). A coherence between two excitonic states is prepared during $T$, whereas the 3PEPS experiments described above generate population states during $T$. Therefore, the photon echo signal along the $T$ axis contains dynamics of quantum mechanical coherence between $\mu$ and $v$, and between $g$ and $\mu$ (or $g$ and $v$ ) along $\tau$ (see Fig. 4). Surprisingly long-lived (440 fs at $77 \mathrm{~K}$ ) electronic coherence between $\mathrm{B}$ and $\mathrm{H}$ was observed in the $R b$. sphaeroides reaction centers with 
the primary electron donor chemically oxidized, suggesting that quantum coherence plays an important role in enhancing the efficiency of energy transfer in pigmentprotein complexes. Lee et al. (2007) argued that correlated protein motion surrounding the pigments is essential to protect the quantum coherence, a mechanism that may be ubiquitous in photosynthetic machinery. The application of the 2CECPE technique to the bacterial reaction center shows how mechanistic details of photosynthetic systems can be obtained using photon echo methods.

\section{Two-dimensional Fourier transform photon echo spectroscopy}

Principles of two-dimensional Fourier transform photon echo spectroscopy

As demonstrated above, photon echo experiments afford control over multiple frequency and time "handles" (i.e., pulse color and duration of time periods $T$ and $\tau$ ). Furthermore, the Fourier relationship between time- and frequency-domain signals is of central importance, and can be exploited by researchers in the design of experiments. Whereas the frequency domain enables observation of excitation energy levels and transition strengths, the time domain allows direct measurement of dynamics. Adopting a time or frequency view accesses different types of information, and the various possibilities underlie the flexibility of photon echo-based techniques. Here we briefly outline the technique of two-dimensional (2D) Fourier transform photon echo spectroscopy, in which a mixed time/frequency view gives a wealth of information about the system of interest.

A 2D spectrum graphs transitions along two frequency axes and contains information about correlation between transitions at different frequencies (Jonas 2003). The direct visualization of correlation, or coupling, in the off-diagonal region of a $2 \mathrm{D}$ spectrum is the method's most immediate advantage over linear spectroscopy. The technique is the most versatile photon echo technique, taking advantage of rephasing to remove inhomogeneous broadening, while presenting both a frequency and time view of the system, allowing simultaneous characterization of the system's energetics and dynamics. This is accomplished by viewing the photon echo signal as a function of the two Fourier frequency axes corresponding to the coherence evolution periods $\tau$ and $t$ for a series of population times, $T$.

\section{Experimental considerations}

Measuring a 2D spectrum requires spectral resolution (measurement of all frequency components) of the photon echo signal (for a detailed treatment of the experimental 2D apparatus, see Brixner et al. 2005). The signal is measured in only one phase-matched direction, and a beam alignment is adopted in which the three excitation beams pass through three corners of a square and the signal propagates in the direction of the fourth corner. The photon echo signal, measured while scanning the coherence time, $\tau$, for a given population time, $T$, is directed into a spectrometer and imaged on a CCD (charge-coupled device) camera. Thus, signal evolution over the echo time $t$ is indirectly measured through its Fourier analog, $\omega_{t}$. Heterodyne detection, performed by interfering the signal with a "local oscillator" pulse, identical to the excitation pulses except attenuated by a neutral density filter, allows measurement of both the amplitude and phase of the signal electric field. The signal field is thus measured as a function of $\tau, T$, and $\omega_{t}$, and Fourier transformation along $\tau$ yields the signal as a function of $\omega_{\tau}, T$, and $\omega_{t}$. The spectrum is displayed (in our convention) with the $\omega_{\tau}$ axis as the abscissa and the $\omega_{t}$ axis as the ordinate, and the evolution of the spectrum with increasing $T$ allows observation of dynamics. In analogy to transient absorption experiments, the $\omega_{\tau}$ axis corresponds to the "pump" frequency, while the $\omega_{t}$ axis corresponds to the "probe" frequency.

\section{Applications}

The experimental and simulated 2D spectra of light-harvesting complex 3 (LH3) from purple bacteria Rhodopseudomonas acidophila, shown in Fig. 5 (Zigmantas et al. 2006), illustrate the general features of a $2 \mathrm{D}$ spectrum. The overall appearance results from the interference of signals from different processes: positive signals arise from stimulated emission or ground-state bleaching (depletion of population in the ground state as a result of excitation), both of which result in more light being emitted. Excited state absorption to yet-higher levels results in less light emitted and thus in negative signals (Brixner 2005). For example, in Fig. 5, positive signals dominate at early population times $(T<1 \mathrm{ps})$, while negative signals dominate at later times. Peaks along the diagonal in early-population-time $2 \mathrm{D}$ spectra match the peaks observed in a linear absorption spectrum. In the LH3 spectra at $T=0$, two strong diagonal bands are observed corresponding to the dominant $\mathrm{Q}_{\mathrm{y}}$ bands in the near-IR spectrum. A cross peak in a $2 \mathrm{D}$ spectrum connecting two diagonal peaks indicates coupling between the two states. When electronic coupling is sufficiently strong relative to the coupling to the bath, quantum coherence may be preserved long enough for observation, giving rise to coherent cross peaks. However, cross peaks may also arise as a result of irreversible, dissipative energy transfer, namely from higher to 
Fig. 5 The experimental and theoretical 2D spectra of the LH3 complex, corresponding to the real part of electric field at $77 \mathrm{~K}$ at population times $T=0 \mathrm{fs}, 20 \mathrm{fs}, 50 \mathrm{fs}, 1 \mathrm{ps}$,

$2 \mathrm{ps}$, and 5 ps. The B800 and B820 peaks appear at

$\left(\omega_{\tau} \sim 12450 \mathrm{~cm}^{-1}\right.$,

$\left.\omega_{t} \sim 12450 \mathrm{~cm}^{-1}\right)$ and

$\left(\omega_{\tau} \sim 12150 \mathrm{~cm}^{-1}\right.$,

$\left.\omega_{t} \sim 12150 \mathrm{~cm}^{-1}\right)$,

respectively, in the $T=0$ spectrum. All spectra are normalized to the absolute maximum; positive features correspond to "more light" and negative to "less light" (Zigmantas et al. 2006)
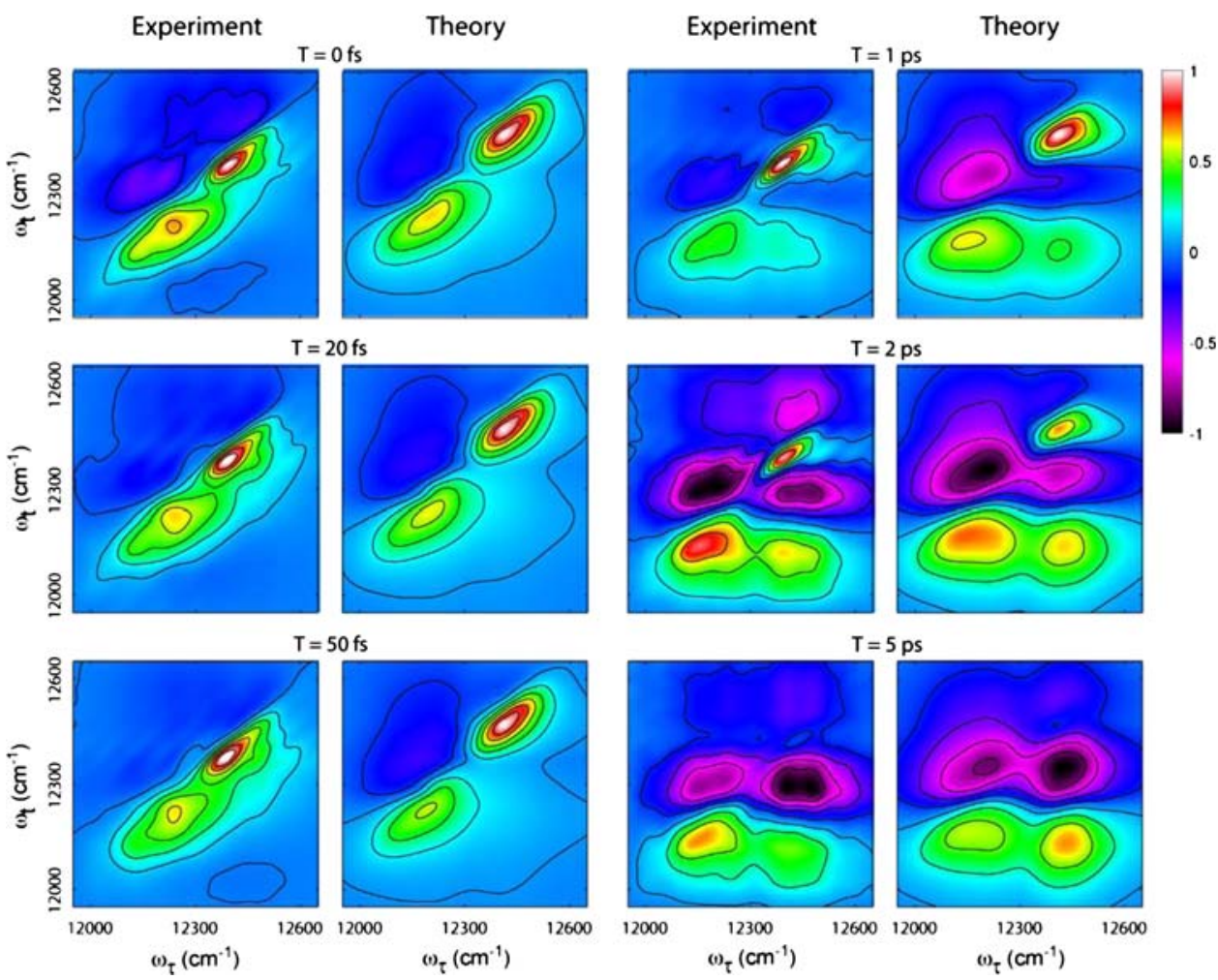

signal above the B800 peak shows that coherence effects are present even for the weakly coupled BChl $a$ ring: if coherent coupling were not present, the B800 peak would be perfectly centered on the diagonal. Thus, 2D spectra are exquisitely sensitive even to weak interactions between chromophores.

The theoretical spectra match the experimental results well, particularly the long-time dynamics in which strong cross peaks appear as energy transfers irreversibly from the B800 to B820 ring. In the $T=1 \mathrm{ps}$ spectrum, a positive cross peak begins to appear, and by 5 ps no population is visible in the B800 band. For a detailed treatment of the theoretical methods used, see Brixner et al. (2004) and Zigmantas et al. (2006). The excellent match between the experimental and simulated spectra demonstrates that the model captures the energy level structure and general dynamical behavior of the LH3 complex. Furthermore, while the experimental spectra provide a wealth of information alone, the theoretical calculations give deeper insight to the energy transfer mechanisms at work. For example, the theoretical calculations showed energy transfer from the B800 band to dark, high-lying energetic states of the B820 band, a mechanism which increases the rate of energy transfer over that predicted by the traditional Förster theory of energy (Scholes and Fleming 2000).

The LH3 results hint at the importance of quantum coherence effects in photosynthetic light harvesting. A 2D experiment can also be devised to probe quantum coherence effects directly, in a manner related to the $2 \mathrm{CECPE}$ the B800 peak remains unchanged. Still, the off-diagonal 
experiment, as first demonstrated by a study of the FMO complex at $77 \mathrm{~K}$ (Engel et al. 2007). In this experiment, 2D spectra are measured with smaller intervals between $T$ time-points, such that rapid oscillations in signal amplitude are sampled. These oscillations result from the reversible, wavelike motion of quantum superposition states. The persistence of the oscillations (longer than $660 \mathrm{fs}$ ) indicates that the coherent nature of the electronic-excited states spanning multiple pigments is maintained for a surprisingly long time after laser excitation, whereas it was assumed previously that vibrational motions would destroy the electronic coherence within $\sim 100$ fs. Figure 6 demonstrates how taking slices through the 2D spectra of FMO from Chlorobium tepidum and lining them up in $T$ reveals the oscillatory motion. The Fourier transform of the oscillations gives a beat spectrum, revealing the energy differences between coupled excitonic states giving rise to the quantum interference. As discussed above in the 2CECPE study of the bacterial reaction center, the quantum mechanical nature of energy transfer may be advantageous for more efficient sampling of a complex energy landscape in photosynthetic systems, as well as for robustness against trapping in local energetic minima.

The above 2D experiments illustrate that key mechanistic information can be extracted from spectra measured using identical input pulses. Experimental strategies involving different polarizations of the laser pulses may be used to probe an increased, or more specific, set of interactions. Such an approach is analogous to linear and circular dichroism methods in one-dimensional spectroscopy (see Garab and Van Amerongen, this issue), except with increased versatility as here four pulses can be independently controlled (Hochstrasser 2001; Zanni et al. 2001; Dreyer et al. 2003). The usefulness of rotating the pulse polarizations lies in the fact that in 2D spectra measured with parallel-polarized input pulses, diagonal peaks dominate the spectra (as evident in the $T=0 \mathrm{LH} 3$ spectrum of Fig. 5), obscuring off-diagonal peaks that report on chromophore interactions. The first example of $2 \mathrm{D}$ electronic spectroscopy using polarization techniques to uncover weak signals was also in an application to the FMO complex (Read et al. 2007). Extending techniques applied in the infrared regime (Hochstrasser 2001; Zanni et al. 2001), the polarization sequence $\left(60^{\circ},-60^{\circ}, 0^{\circ}, 0^{\circ}\right)$ for pulses $(1$, $2,3, \mathrm{LO})$ was used to completely eliminate the diagonal peaks from the spectrum of FMO from Pelodictyon phaeum (Fig. 7).

In addition to highlighting otherwise weak features, polarization techniques can be used to report on structures of photosynthetic complexes. The amplitude of a cross peak in a 2D spectrum, and the way that the amplitude changes with input pulse polarization, depends in part on the relative orientation between the coupled transition dipoles. In a measurement on the FMO complex from Prosthecochloris aestuarii using one set of pulse polarizations, a cross peak is only weakly visible in the spectrum at $400 \mathrm{fs}$ (Fig. $8\left(45^{\circ},-45^{\circ}, 0^{\circ}, 0^{\circ}\right)$ spectrum), while under another polarization scheme (Fig. $8\left(75^{\circ},-75^{\circ}, 0^{\circ}, 0^{\circ}\right)$ spectrum) the cross peak appears strongly (Read et al. 2008). Based on these data in combination with theoretical calculations, we concluded that the relative angle between the two dominant transition dipoles in FMO is roughly $40^{\circ}$.

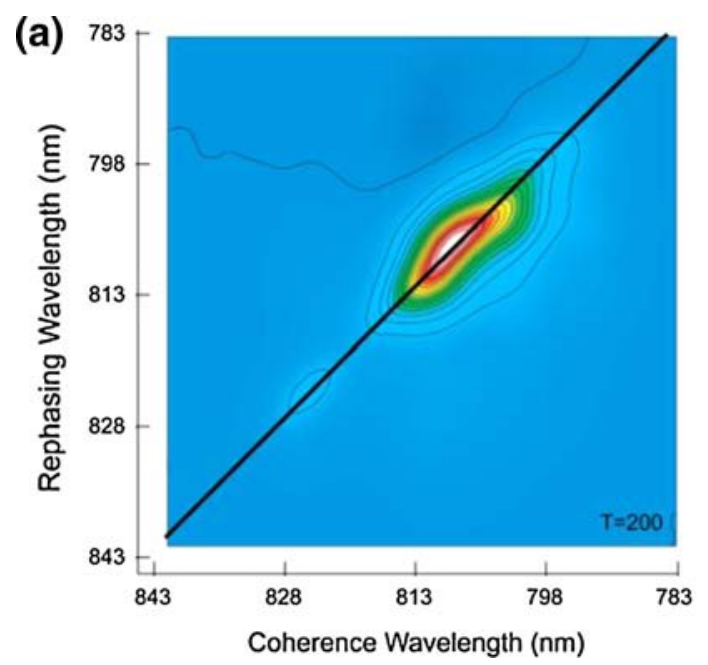

Fig. 6 Electronic coherence beating: a representative 2D spectrum is shown in panel a with a line across the main diagonal peak. The amplitude along this diagonal line is plotted against population time in panel $\mathbf{b}$ with a black line covering the exciton 1 peak amplitude; the data is scaled by a smooth function effectively normalizing the data without affecting oscillations. A spline interpolation is used to

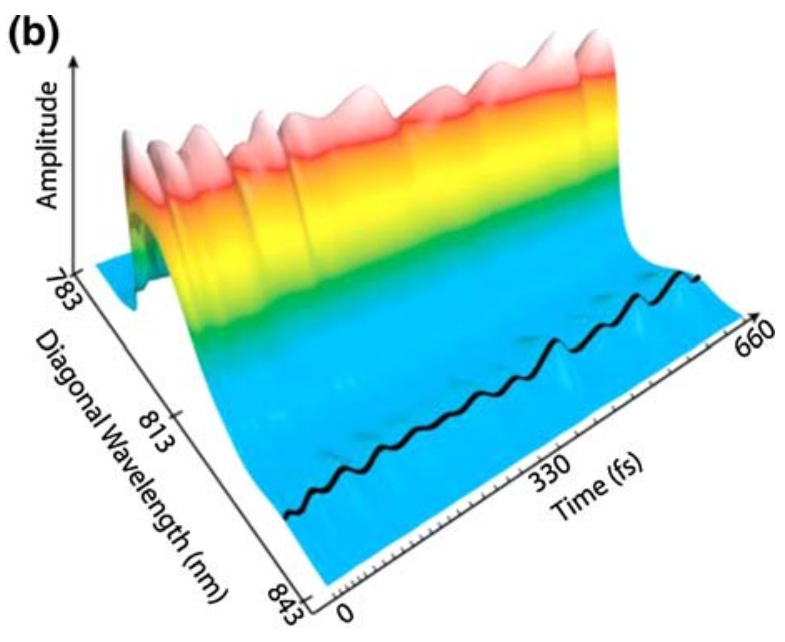

connect the spectra; the times at which spectra were taken are denoted by tick marks along the time axis. The oscillatory black curve shows coherent quantum beating of exciton 1. Figure reprinted with permission from Macmillan Publishers Ltd: Engel et al. (2007); Copyright 2007 


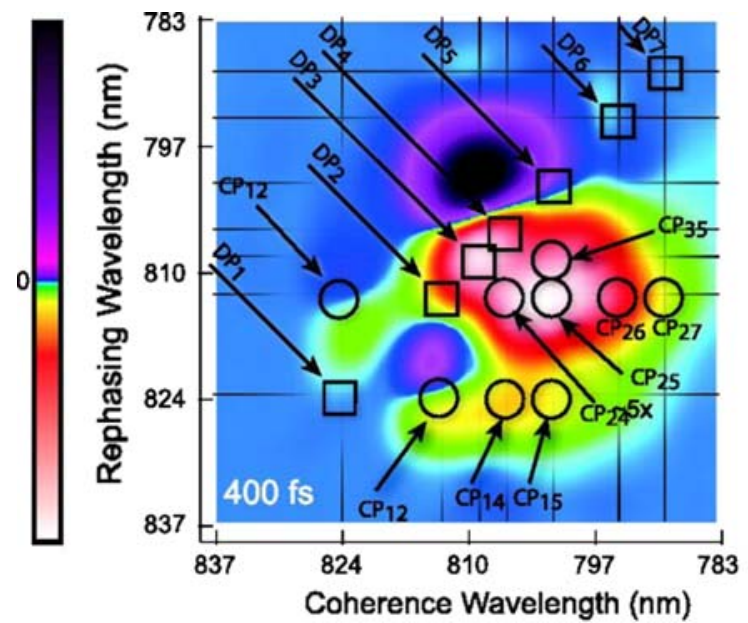

Fig. 7 The cross peak specific 2D and conventional 2D electronic spectra for FMO from Pelodictyon phaeum. The cross-peak specific spectrum reveals off-diagonal features obscured by the diagonal peaks in the conventional 2D spectrum. Both spectra are colored using a nonlinear ArcSinh coloration to emphasize smaller features, and the cross-peak specific coloration is inverted to facilitate direct visual comparison of the cross peaks to those in the conventional 2D
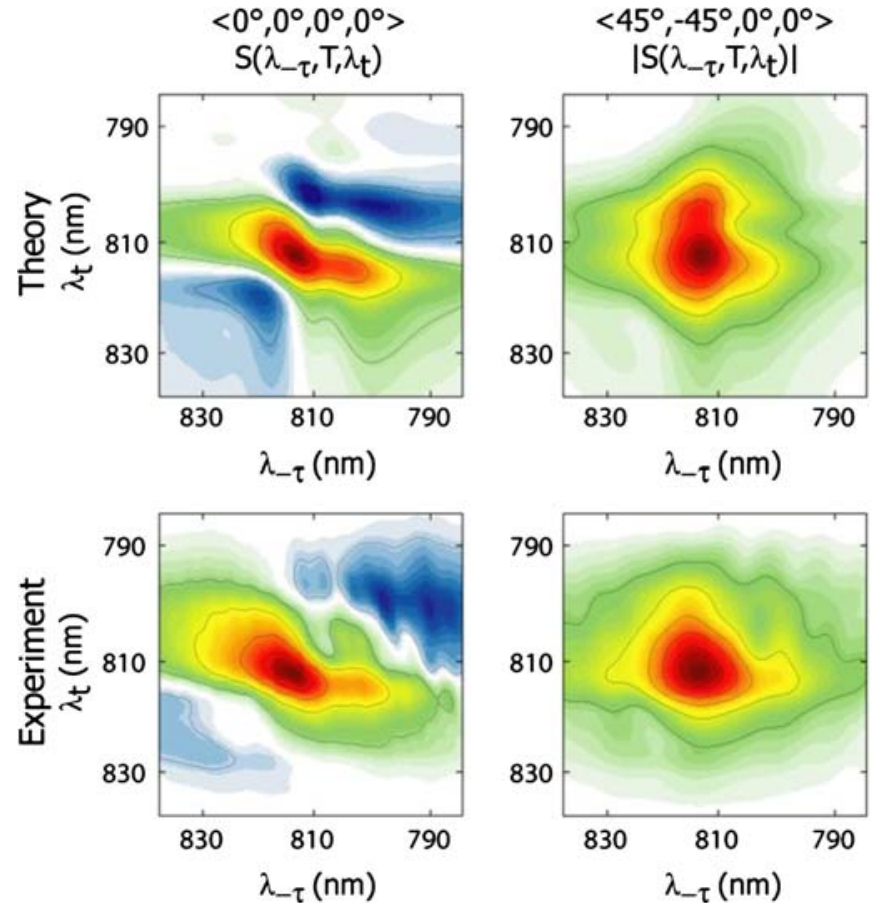

Fig. 8 Theoretical and experimental spectra of FMO from Prosthecochloris aestuarii at $T=400 \mathrm{fs}$ and $77 \mathrm{~K}$ (nonrephasing part, for details, see Read et al. 2008). Top row, left to right: theoretical $<0^{\circ}$, $0^{\circ}, 0^{\circ}, 0^{\circ}>,<45^{\circ},-45^{\circ}, 0^{\circ}, 0^{\circ}>$, and $<75^{\circ},-75^{\circ}, 0^{\circ}, 0^{\circ}>2 \mathrm{D}$ spectra. The top right panel shows experimental and theoretical linear absorption spectra in black and red, respectively, and the dotted line is the laser spectrum of the pulses used to measure $2 \mathrm{D}$ spectra. Bottom

The experiment and corresponding theoretical calculations point to the way that $2 \mathrm{D}$ experiments can be designed to probe particular interactions in a multi-chromophore

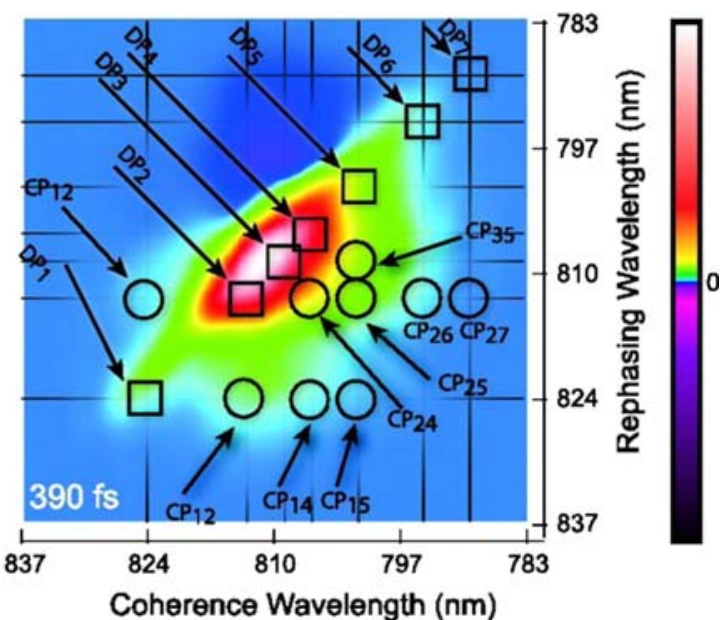

spectrum. Diagonal peaks $\left(\mathrm{DP}_{i}\right)$ are shown with squares while cross peaks $\left(\mathrm{CP}_{i j}\right)$ are denoted with circles. The shape of the edge of the cross peak regions agrees between the spectra, but significant additional structure is visible in the cross peak specific spectrum. Figure from Read et al. (2007); Copyright 2007, National Academy of Sciences, USA
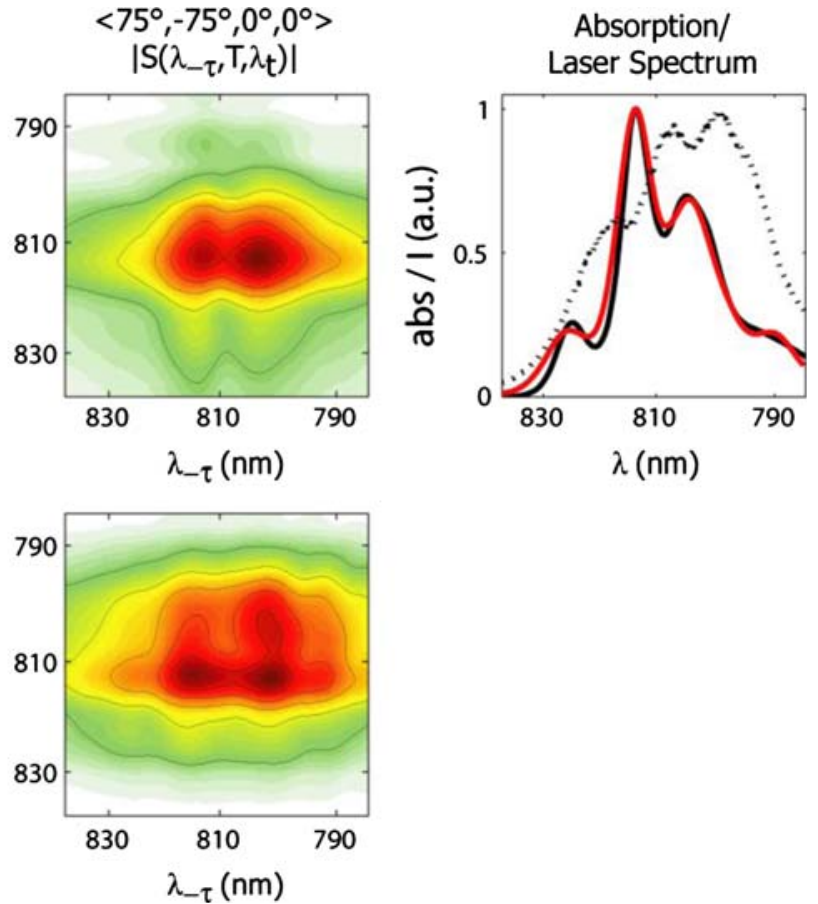

row, left to right: experimental $\left.<0^{\circ}, 0^{\circ}, 0^{\circ}, 0^{\circ}\right\rangle,<45^{\circ},-45^{\circ}, 0^{\circ}, 0^{\circ}>$, and $<75^{\circ},-75^{\circ}, 0^{\circ}, 0^{\circ}>2 \mathrm{D}$ spectra. The differently polarized spectra show different cross peak amplitudes. In particular, a strong cross peak visible in the $<75^{\circ},-75^{\circ}, 0^{\circ}, 0^{\circ}>$ spectrum is absent from the $<45^{\circ},-45^{\circ}, 0^{\circ}, 0^{\circ}>$ spectrum. Figure reprinted with permission from Biophysical Society, Read et al. (2008); Copyright 2008

system and thus yield detailed quantitative insight on the coupling strengths and relative orientations between transition dipole moments. 


\section{Conclusions}

In summary, photon echo-based experiments may be designed to probe a number of aspects of photosynthetic light-harvesting complexes in detail, including coupling among pigments, coupling between pigments and the surrounding protein environment, contributions to spectral broadening, dynamical time scales, and mechanisms of energy transfer in light harvesting. Perhaps most exciting at this juncture is the recently realized capability of photon echo techniques to directly probe the quantum mechanical underpinnings of ultrafast energy transfer in photosynthesis, first discussed over 50 years ago (see review by Knox 1996), but elusive of direct experimental observation until now. The experiments described above demonstrate some of the experimental techniques that can be utilized to probe various aspects of light harvesting in detail. However, the flexibility of photon echo techniques means that a myriad of different experiments could be devised in addition to those outlined here in this review. From an experimental standpoint, the technological implementation of photon echo experiments is still in an early phase. While routine generation of sub-100 fs pulses has now been achieved, phase detection and control still present a problem for programmable pulse sequences, which would significantly aid in widespread applicability of these techniques. Thus, coming years will likely see rapid expansion of experimental methods related to those described here, and much is to be gained in our understanding of photosynthetic light harvesting from such developments.

Acknowledgements This work was supported by the Director, Office of Science, Office of Basic Energy Sciences, of the U.S. Department of Energy under Contract No. DE-AC02-05CH11231 and by the Chemical Sciences, Geosciences and Biosciences Division, Office of Basic Energy Sciences, U.S. Department of Energy under contract DE-AC03-76SF000098. This manuscript was edited by Govindjee.

Open Access This article is distributed under the terms of the Creative Commons Attribution Noncommercial License which permits any noncommercial use, distribution, and reproduction in any medium, provided the original author(s) and source are credited.

\section{References}

Blankenship RE (2002) Molecular mechanisms of photosynthesis. Blackwell Science, Oxford

Brixner T, Mancal T, Stiopkin IV, Fleming GR (2004) Phasestabilized two-dimensional electronic spectroscopy. J Chem Phys 121:4221-4236

Brixner T, Stenger J, Vaswani HM, Cho M, Blankenship RE, Fleming GR (2005) Two-dimensional spectroscopy of electronic couplings in photosynthesis. Nature 434:625-658
Bruggemann B, Kjellberg P, Pullerits T (2007) Non-perturbative calculation of 2D spectra in heterogeneous systems: Exciton relaxation in the FMO complex. Chem Phys Lett 444:192-196

Cho M, Yu JY, Joo TH, Nagasawa Y, Passino SA, Fleming GR (1996) The integrated photon echo and solvation dynamics. J Phys Chem 100:11944-11953

Christensson N, Dietzek B, Pascher T, Yartsev A, Pullerits T (2008) Three-pulse photon echo peak shift in optically dense samples. Chem Phys Lett 457:106-109

Demtroder W (2003) Laser spectroscopy, 3rd edn. Springer, Berlin

Dreyer J, Moran AM, Mukamel S (2003) Tensor components in three pulse vibrational echoes of a rigid dipeptide. Bull Kor Chem Soc 24:1091-1096

Engel GS, Calhoun TR, Read EL, Ahn TK, Mancal T, Cheng YC, Blankenship RE, Fleming GR (2007) Evidence for wavelike energy transfer through quantum coherence in photosynthetic systems. Nature 446:782-786

Fleming GR, Cho M (1996) Chromophore-solvent dynamics. Annu Rev Phys Chem 47:109-134

Garab G, Van Amerongen $H$ (this issue) Linear dichroism and circular dichroism in photosynthesis research. Photosynth Res. doi:10.1007/s11120-009-9424-4

Hochstrasser RM (2001) Two-dimensional IR-spectroscopy: polarization anisotropy effects. Chem Phys 266:273-284

Jimenez R, Fleming GR (1996) Ultrafast spectroscopy of photosynthetic systems. In: Amesz J, Hoff AJ (eds) Biophysical techniques in photosynthesis. Advances in photosynthesis and respiration, vol 3. Springer, Dordrecht, pp 63-73

Jimenez R, Van Mourik F, Yu JY, Fleming GR (1997) Three-pulse photon echo measurements on LH1 and LH2 complexes of Rhodobacter sphaeroides: a nonlinear spectroscopic probe of energy transfer. J Phys Chem B 101:7350-7359

Jonas DM (2003) Two-dimensional femtosecond spectroscopy. Annu Rev Phys Chem 54:425-463

Knox RS (1996) Electronic excitation transfer in the photosynthetic unit: reflections on work of William Arnold. Photosynth Res 48:35-39

Lee H, Cheng YC, Fleming GR (2007) Coherence dynamics in photosynthesis: protein protection of excitonic coherence. Science 316:1462-1465

Mancal T, Fleming GR (2004) Probing electronic coupling in excitonically coupled heterodimer complexes by two-color three-pulse photon echoes. J Chem Phys 121:10556-10565

Mukamel S (1995) Principles of nonlinear optical spectroscopy. Oxford University Press, New York

Parkinson DY, Lee H, Fleming GR (2007) Measuring electronic coupling in the reaction center of purple photosynthetic bacteria by two-color, three-pulse photon echo peak shift spectroscopy. J Phys Chem B 111:7449-7456

Parson WW (2007) Modern optical spectroscopy. Springer, Berlin

Read EL, Engel GS, Calhoun TR, Ahn TK, Mancal T, Cheng YC, Blankenship RE, Fleming GR (2007) Cross-peak-specific twodimensional electronic spectroscopy. Proc Natl Acad Sci USA 104:14203-14208

Read EL, Schlau-Cohen GS, Engel GS, Wen JZ, Blankenship RE, Fleming GR (2008) Visualization of excitonic structure in the Fenna-Matthews-Olson photosynthetic complex by polarization-dependent two-dimensional electronic spectroscopy. Biophys J 95:847-856

Rulliere C (ed) (2003) Femtosecond laser pulses: principles and experiments, 2nd edn. Springer, USA

Scholes GD, Fleming GR (2000) On the mechanism of light harvesting in photosynthetic purple bacteria: B800 to B850 energy transfer. J Phys Chem B 104:1854-1868

Van Amerongen H, Valkunas L, Van Grondelle R (2000) Photosynthetic excitons. World Scientific, Singapore 
Yu JY, Nagasawa Y, Van Grondelle R, Fleming GR (1997) Three pulse echo peak shift measurements on the B820 subunit of LH1 of Rhodospirillum rubrum. Chem Phys Lett 208: 404-410

Zanni MT, Ge NH, Kim YS et al (2001) Two-dimensional IR spectroscopy can be designed to eliminate the diagonal peaks and expose only the crosspeaks needed for structure determination. Proc Natl Acad Sci USA 98:11265-11270

Zigmantas D, Read EL, Mancal T, Brixner T, Gardiner AT, Cogdell RJ, Fleming GR (2006) Two-dimensional electronic spectroscopy of the B800-B820 light-harvesting complex. Proc Natl Acad Sci USA 103:12672-12677 